\title{
Control of an Inverted Wheeled Pendulum on a Soft Surface
}

\section{O. M. Kiselev}

The dynamics of an inverted wheeled pendulum controlled by a proportional plus integral plus derivative action controller in various cases is investigated. The properties of trajectories are studied for a pendulum stabilized on a horizontal line, an inclined straight line and on a soft horizontal line. Oscillation regions on phase portraits of dynamical systems are shown. In particular, an analysis is made of the stabilization of the pendulum on a soft surface, modeled by a differential inclusion. It is shown that there exist trajectories tending to a semistable equilibrium position in the adopted mathematical model. However, in numerical simulations, as well as in the case of real robotic devices, such trajectories turn into a limit cycle due to round-off errors and perturbations not taken into account in the model.

Keywords: pendulum, control, stability, differential inclusion

\section{Introduction}

An inverted wheeled pendulum is a popular model used to investigate the dynamics and control systems of robotic devices.

This paper derives dynamics equations for a wheeled pendulum moving uniformly on a soft surface. Analysis is made of degenerations of these equations for motion on a rough surface, in a horizontal straight line and in a straight line of constant inclination under the action of a proportional plus derivative action controller (PD controller) and under the action of a proportional plus integral plus derivative action controller (PID controller). The properties of the phase space of corresponding dynamical systems are investigated and regions are obtained in which the inverted pendulum is stabilized by a PD controller. It is shown how the the region of stable motion of the pendulum diminishes as the inclination of the surface is varied. The stability conditions are considered in bounded regions for initial nonlinear equations.

Received December 16, 2019

Accepted May 19, 2020

\author{
Oleg M. Kiselev \\ ok@ufanet.ru \\ Institute of Mathematics with Computing Centre - \\ Subdivision of the Ufa Federal Research Centre of the Russian Academy of Science \\ ul. Chernyshevskogo 112, Ufa, 450008 Russia
}


The motion of an inverted wheeled pendulum on a soft surface in the presence of rolling friction is examined. It is shown that there exist conditions under which the wheeled pendulum stabilizes during accelerated motion of the wheel. Generally speaking, this mode causes the pendulum to accelerate and leads to the loss of stabilization due to the fact that the dynamical performance of the motor is limited. It is shown that the introduction of an integral component into the PD controller leads to the onset of a limit cycle and, as a consequence, to the stabilization of motions in the neighborhood of such a cycle.

In recent years, there has been an extensive amount of research devoted to an inverted wheeled pendulum. A historical review and a consecutive statement of problems concerning the control of the pendulum in various devices can be found in the monographs [1,2]. A brief review of publications discussing a control system for the inverted wheeled pendulum is given, for example, in [3].

A derivation of equations for a wheeled pendulum and the problem of synthesis of control is discussed, for example, in the book [4] and in the paper [5]. Problems of the stability and control of an inverted pendulum on a two-wheeled platform were addressed, for example, in [6-9] for a pendulum on an inclined surface. The problem of negotiating vertical obstacles was addressed in [10]. The motion of a robot on a rough surface was studied in [11], and the stability of motion on a rough surface was analyzed in [12].

PID controllers are widely used in technical devices (see, e.g., [13]). General approaches to the control of models defined by linear second-order systems were considered, for example, in [14] from the viewpoint of analysis of the evolution of first integrals. PD and PID controllers are rather often used to control two-wheeled robots (see, e.g., $[15,16])$ and the literature review [3].

To solve the problem of stabilizing the pendulum in the upper position, one usually considers either linearization in a small neighborhood of the upper point of the pendulum, as in most of the above-mentioned publications, or, for example, nonsmooth control based of the Lyapunov function for the inverted wheeled pendulum [17].

The results of the above-mentioned studies are supplemented according to the general view of the wheeled pendulum as a dynamical system. First integrals are found which appear when a proportional controller is used. The domains of attraction of steady-state solutions for a PD controller are investigated using suitable Lyapunov functions, which are obtained from conservation laws taking into account the differential part of the controller.

The paper is structured as follows. Section 2 derives equations of motion for a wheeled pendulum using the Lagrangian function. In general this is a standard approach, however, here this derivation is presented for general understanding of how the parameters of the nondimensionalized system of equations are related to the initial mechanical system - the wheeled pendulum.

Section 3 discusses the motion of the pendulum on a plane curve of an arbitrary shape without control action.

Section 4 addresses the problem of stabilizing the pendulum under the action of a PD controller on a smooth surface. An equation of motion is derived and it is shown that the upper equilibrium point is asymptotically stable.

Section 5 is concerned with the dynamics of the pendulum's motion in a straight line with constant inclination. It is shown that a PD controller with a constantly acting torque is necessary to ensure the asymptotic stability of the pendulum under the condition of unaccelerated rotation of the wheel.

Section 6 examines the motion on a soft surface. To set the pendulum in motion, it is necessary to overcome the rolling friction torque. The rolling friction torque leads to the appearance of the discontinuous coefficient in the system of differential equations. A hypersurface arises in 
the phase space on which the value of the discontinuous coefficient is undefined. In this case, it is convenient to model the mechanical system by means of differential inclusion. The use of differential inclusions in the problem with dry friction is described, for example, in the book [18].

It has turned out that it is not possible to stabilize the upper point of the pendulum using a PID controller when it is located on a soft horizontal line. However, a stable limit cycle arises in the neighborhood of the upper position of the pendulum. As a result, dynamical stabilization occurs due to the limit cycle located in the neighborhood of the unstable point of the pendulum.

\section{Equations of motion for a wheeled pendulum}

We consider equations of motion for the mechanical structure of a wheeled pendulum.

Let $m$ be the mass of the pendulum shown in Fig. 1 and $M$ the mass of the rim of the wheel. Assume that the pendulum is acted upon by gravity directed vertically downward with free-fall acceleration $g$.

We consider motion along the curve $u(\beta), v(\beta)$, where the coordinate $u$ is the horizontal coordinate of the curve and $v$ is the vertical coordinate of the curve along which the wheel moves.

If we assume that $\alpha$ is the angle of deviation of the pendulum from the vertical and $\beta$ is the angle of rotation of the wheel, then the kinetic energy is

$$
T=\frac{m}{2}\left(\dot{x}^{2}+\dot{y}^{2}\right)+\frac{M}{2}\left(r^{2} \dot{\beta}^{2}+\dot{u}^{2}+\dot{v}^{2}\right) .
$$

The potential energy for the mechanical structure of the wheeled pendulum shown in Fig. 1 is

$$
\Pi=g v M+g m y .
$$

We use the generalized coordinates $\alpha, \beta$ with

$$
\begin{aligned}
x=l \sin (\alpha)+u, & y=l \cos (\alpha)+v, \\
u=u(\beta), & v=v(\beta), \\
\dot{u}=r \dot{\beta} \cos (z), & \dot{v}=r \dot{\beta} \sin (z) .
\end{aligned}
$$

Here $z(\beta)$ is a given function defining the inclination of the curve $u(\beta), v(\beta)$.

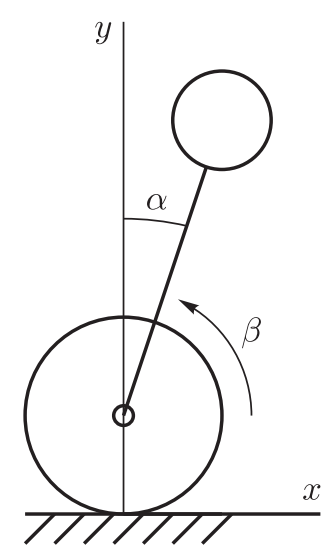

Fig. 1. A wheeled pendulum. Let $r$ denote the radius of the wheel, $l$ the length of the pendulum, $\alpha$ the angle of deviation of the pendulum from the vertical, and $\beta$ the angle of rotation of the wheel relative to some initial position. We assume that the pendulum is fastened with a hinge on the wheel's axis in such a way that it can freely rotate relative to the wheel. 
As a result, one can obtain a Lagrangian function depending on two variables $\alpha, \beta$ and the given curve $z(\beta)$, which defines the coordinate of the supporting point of the wheel.

The Lagrange equations rewritten for the coordinates $\alpha, \beta$ are a system of two second-order equations:

$$
\frac{d}{d t}\left(\frac{\partial L}{\partial \dot{\alpha}}\right)-\frac{\partial L}{\partial \alpha}=0, \quad \frac{d}{d t}\left(\frac{\partial L}{\partial \dot{\beta}}\right)-\frac{\partial L}{\partial \beta}=0 .
$$

Before investigating the explicit form of this system, it is convenient to introduce the following notation:

$$
\rho=\frac{r}{l}, \quad \zeta=\frac{m}{M}, \quad \tau=\sqrt{\frac{g}{l}} t, \quad \frac{d u}{d \beta}=\cos (z), \quad \frac{d v}{d \beta}=\sin (z) .
$$

To simplify the notation, we will use a dot and a prime to denote the derivative with respect to the independent variable $\tau$ and the parameter $\beta$, respectively:

$$
\frac{d f}{d \tau} \equiv \dot{f}, \quad \frac{d g}{d \beta} \equiv g^{\prime}
$$

Then we obtain a system of equations defining the angles of rotation:

$$
\begin{aligned}
\ddot{\alpha} & =\sin (\alpha)-\left(\cos (\alpha-z) \ddot{\beta}+\sin (\alpha-z) z^{\prime} \dot{\beta}^{2}\right) \rho, \\
(\zeta+2) \rho \ddot{\beta} & =-\sin (z)-\left(\ddot{\alpha} \cos (\alpha-z)-\dot{\alpha}^{2} \sin (\alpha-z)\right) \zeta .
\end{aligned}
$$

Here $z(\beta)$ is a given function defining the surface on which the wheel rolls.

This system of equations degenerates to an equation of the mathematical pendulum if the radius of the wheel is taken to be zero with $\rho=0$ :

$$
\ddot{\alpha}=\sin (\alpha) .
$$

If the wheeled pendulum is on the horizontal surface, i.e., $z \equiv 0$, then one can decouple from the system one equation for $\alpha$ :

$$
\left(\sin ^{2}(\alpha) \zeta+2\right) \ddot{\alpha}=(\zeta+2) \sin (\alpha)-\frac{1}{2} \dot{\alpha}^{2} \zeta \sin (2 \alpha) .
$$

For a pendulum on a surface with constant inclination $z \equiv \varepsilon$ one can also decouple one second-order equation for the angle of rotation of the pendulum $\alpha$ :

$$
\left(\sin ^{2}(\epsilon-\alpha) \zeta+2\right) \ddot{\alpha}=(2+\zeta) \sin (\alpha)+\sin (\epsilon) \cos (\epsilon-\alpha)+\frac{1}{2} \zeta \dot{\alpha}^{2} \sin (2(\epsilon-\alpha)) .
$$

Equations (2.2)-(2.4) are integrable with corresponding conservation laws:

$$
\begin{aligned}
E & =\frac{\dot{\alpha}^{2}}{2}+\cos (\alpha), \\
E_{0} & =\dot{\alpha}^{2}+(\zeta+2) \cos (\alpha)+\frac{1}{2} \dot{\alpha}^{2} \sin ^{2}(\alpha) \zeta, \\
E_{\epsilon} & =\dot{\alpha}^{2}+(\zeta+2) \cos (\alpha)+\sin (\epsilon) \sin (\epsilon-\alpha)+\frac{1}{2} \dot{\alpha}^{2} \sin ^{2}(\epsilon-\alpha) \zeta .
\end{aligned}
$$

Here $E, E_{0}$ and $E_{\epsilon}$ are conservation laws for (2.2), (2.3) and (2.4), respectively. 


\section{Stationary points for a wheeled pendulum}

Here and in what follows, by steady-state solutions we mean those solutions of the system of equations (2.1) for which $\alpha=$ const.

In particular, for a pendulum on a smooth supporting surface (2.3) the stationary point $\alpha_{s}=0$ corresponds to a saddle, i.e., such a point is unstable, and

$$
\beta=\beta_{1} \tau+\beta_{0}
$$

for $\forall \beta_{1}, \beta_{0} \in \mathbb{R}$. Thus, the wheeled pendulum can move with constant velocity.

For a pendulum on a surface with constant inclination the equation for a stationary point is

$$
(2+\zeta) \sin \left(\alpha_{s}\right)+\sin (\epsilon) \cos \left(\epsilon-\alpha_{s}\right)=0 .
$$

The solution is

$$
\alpha_{s}=\arctan \left(\frac{\sin (2 \varepsilon)}{2\left(2+\zeta+\sin ^{2}(\epsilon)\right)}\right) .
$$

In this position the whole wheeled pendulum rolls on the surface with constant inclination and the wheel rotates with constant angular acceleration:

$$
\ddot{\beta}_{s}=\frac{-\sin (\epsilon)}{(\zeta+2) \rho}, \quad \text { or } \quad \beta=\frac{-\sin (\epsilon)}{2(\zeta+2) \rho} \tau^{2}+\beta_{1} \tau+\beta_{0}
$$

In the general position case, the stabilization of the position of the pendulum leads to a redefined system of equations for the wheel's angle of rotation $\beta(\tau)$ and $\alpha_{s}=$ const:

$$
\begin{gathered}
0=\sin \left(\alpha_{s}\right)-\left(\cos \left(\alpha_{s}-z\right) \ddot{\beta}+\sin \left(\alpha_{s}-z\right) z^{\prime} \dot{\beta}^{2}\right) \rho, \\
(\zeta+2) \rho \ddot{\beta}=-\sin (z) .
\end{gathered}
$$

The condition for existence of a joint solution for this redefined system of equations leads to a second-order equation for the curve $z(\beta)$ :

$$
\begin{gathered}
z^{\prime} \sin \left(\alpha_{s}-z\right)\left(\frac{d}{d \beta}\left(\cos \left(\alpha_{s}-z\right) \frac{\sin (z)}{\zeta+2}\right)+2 z^{\prime} \sin \left(\alpha_{s}-z\right) \frac{\sin (z)}{\zeta+2}\right)= \\
\left(\sin \left(\alpha_{s}\right)+\cos \left(\alpha_{s}-z\right) \frac{\sin (z)}{\zeta+2}\right) \frac{d}{d \beta}\left(z^{\prime} \sin \left(\alpha_{s}-z\right)\right) .
\end{gathered}
$$

\section{Stabilization of a wheeled pendulum on a smooth surface}

Stationary points corresponding to the upper position of the pendulum are unstable. To stabilize the pendulum in a neighborhood of an unstable stationary point, the control of the wheel's rotation is used.

Let the torque of the wheel be $\mu$. If this torque is denoted as $\mu=2 M r h$, then the system of equations for the wheeled pendulum takes the form

$$
\begin{aligned}
\ddot{\alpha} & =\sin (\alpha)-\left(\cos (\alpha-z) \ddot{\beta}-\sin (\alpha-z) z^{\prime} \dot{\beta}^{2}\right) \rho-2 \frac{\rho}{\zeta} h, \\
(\zeta+2) \rho \ddot{\beta} & =-\sin (z)-\left(\ddot{\alpha} \cos (\alpha-z)-\dot{\alpha}^{2} \sin (\alpha-z)\right) \zeta+\frac{2}{\rho} h .
\end{aligned}
$$


In particular, for a wheeled pendulum on a smooth surface $(z \equiv 0)$ we have

$$
\left(\sin ^{2}(\alpha) \zeta+2\right) \ddot{\alpha}=(\zeta+2) \sin (\alpha)-\frac{1}{2} \dot{\alpha}^{2} \zeta \sin (2 \alpha)-2\left(\frac{1}{\rho} \cos (\alpha)+\left(1+\frac{2}{\zeta}\right) \rho\right) h .
$$

The problem of controllability and stability for linearization of the system of equations (4.1) and for linearization of Eq. (4.2) in the neighborhood of $\alpha=0$ is addressed, for example, in the book [4] (Chapter $1 \S 2$ ). The approach to investigating the stability by linearization is a standard approach used to construct control systems for nonlinear systems in control theory and engineering applications. Below we consider the stability of a feedback system for the nonlinear system (4.1) and the nonlinear equation (4.2).

Consider stabilization using a PD controller (see, e.g., [16] and the review [3]):

$$
h=k_{1} \alpha+k_{2} \dot{\alpha} .
$$

If $k_{2}=0$, the equation for the wheeled pendulum on the smooth surface with control turns out to be integrable. The integral of motion for this equation is

$$
\mathcal{E}_{0}=\left(1+\frac{1}{2} \sin ^{2}(\alpha) \zeta\right) \dot{\alpha}^{2}+(\zeta+2) \cos (\alpha)+k_{1}\left(\frac{2}{\rho}\left(\alpha \sin (\alpha)+\cos (\alpha)+\alpha^{2} \rho\left(1+\frac{2}{\zeta}\right)\right) .\right.
$$

The stationary points $\alpha_{i}$ for the pendulum with control are defined by the equation

$$
2 k_{1} \rho^{2}(\zeta+2) \alpha-\zeta \rho(\zeta+2) \sin (\alpha)+2 k_{1} \zeta \alpha \cos (\alpha)=0 .
$$

When

$$
k_{1}<\frac{\zeta \rho(2+\zeta)}{2 \zeta+2 \rho^{2}(2+\zeta)}
$$

there exists the only stationary point $\alpha_{0}=0$, which is a saddle.

When

$$
k_{1}>\frac{\zeta \rho(2+\zeta)}{2 \zeta+2 \rho^{2}(2+\zeta)}
$$

there exist three stationary points: $\alpha_{0}$, which is a stable focus if $k_{2}=0$ is a center; the other two points $\alpha_{1}>0, \alpha_{2}<0$ are saddles.

In this case, there exist two heteroclinic curves (separatrices), one of which emanates from the saddle $\alpha_{1}$ and tends to the saddle $\alpha_{2}$ as $\tau \rightarrow \infty$, and the other emanates from the saddle $\alpha_{2}$ and tends to $\alpha_{1}$ as $\tau \rightarrow \infty$. These separatrices bound the oscillation zone around the point $\alpha_{0}$ (see Fig. 2).

On the separatrices, the value of the conservation law is

$$
\mathcal{E}_{0}^{(s)}=(\zeta+2) \cos \left(\alpha_{1}\right)+k_{1}\left(\frac{2}{\rho}\left(\alpha_{1} \sin \left(\alpha_{1}\right)+\cos \left(\alpha_{1}\right)+\alpha_{1}^{2} \rho\left(1+\frac{2}{\zeta}\right)\right) .\right.
$$

Thus, the separatrices are defined by the curves on the phase plane $(\alpha, \dot{\alpha})$ by the given value of $\mathcal{E}_{0}^{(s)}$. An example is given in Fig. 2 .

When $k_{2}>0$, the derivative $\mathcal{E}_{0}$ has the following form by virtue of Eq. (4.2):

$$
\dot{\mathcal{E}}_{0}=-2 k_{2} \dot{\alpha}^{2}\left(\left(1+\frac{2}{\zeta}\right) \rho+\frac{1}{\rho} \cos (\alpha)\right) .
$$

It follows from this formula that the point $(0,0)$ is a stable focus. When $k_{2}>0$, all trajectories from the oscillation zone tend to it. When $(\alpha, \dot{\alpha})=(0,0)$, it follows from the second equation of (2.1) that $\ddot{\beta}=0$, that is, the wheel can rotate with constant velocity. 


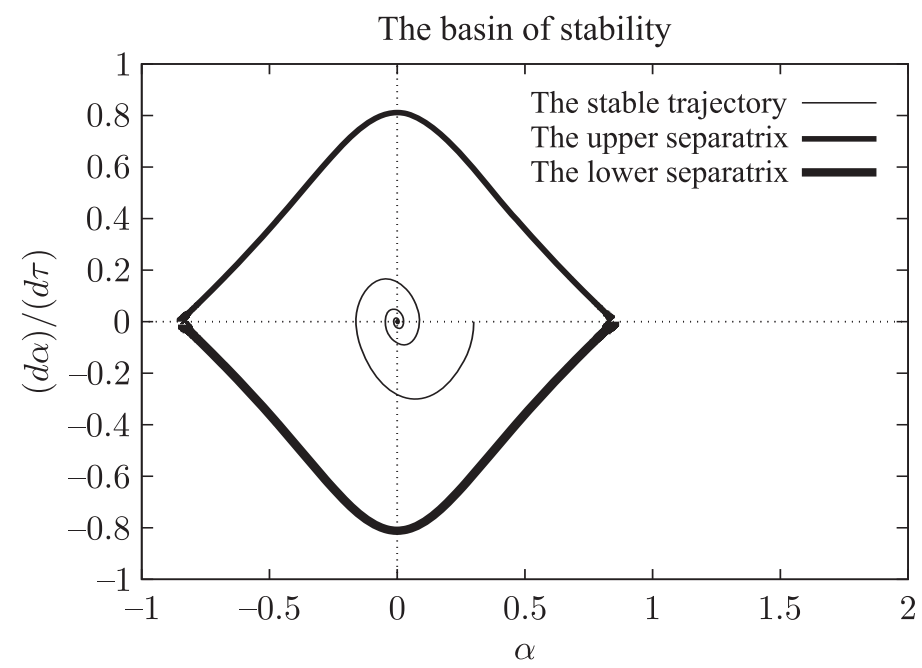

Fig. 2. Phase portrait for Eq. (4.2) in a neighborhood of the stationary point with $\zeta=10, \rho=0.2$, $k_{1}=1.5, k_{2}=0.05$. The upper and lower separatrices (for which $k_{2}=0$ ) bound the domain of attraction of the trajectories of the stationary point $(0,0)$. The curve tending to the point $(0,0)$ has been obtained by numerically solving Eq. (4.2) using the Runge-Kutta fourth-order method.

The result of this section is

Theorem 1. If condition (4.3) is satisfied, the trajectories of Eq. (2.3) inside the region bounded by the separatrices defined by Eq. (4.4) tend, as $t \rightarrow \infty$, to the point $(0,0)$ on the phase plane $(\alpha, \dot{\alpha})$ provided that $k_{2}>0$. At the point $(\alpha, \dot{\alpha})=(0,0)$ the acceleration of the wheel is $\ddot{\beta}=0$.

\section{Stabilization on a surface with constant inclination}

For a pendulum on a surface with constant inclination $z \equiv \epsilon=$ const the equation for $\alpha$ has the form

$$
\begin{gathered}
\left(\sin ^{2}(\epsilon-\alpha) \zeta+2\right) \ddot{\alpha}=(2+\zeta) \sin (\alpha)+\sin (\epsilon) \cos (\epsilon-\alpha)+ \\
\frac{1}{2} \zeta \dot{\alpha}^{2} \sin (2(\epsilon-\alpha))-2\left(\frac{1}{\rho} \cos (\epsilon-\alpha)+\left(1+\frac{2}{\zeta}\right) \rho\right) h .
\end{gathered}
$$

In Section 3 it is shown that the stabilization of the pendulum without control action leads to uniformly accelerated motion of the wheel on an inclined surface. Such a mode does not satisfy the problem of stabilizing the wheeled pendulum since the wheel itself accelerates continuously.

Linearized equations of motion for systems on an inclined surface which are similar in design are discussed, for example, in [9], see also [7]. Below we consider the behavior of the wheeled pendulum in a region where nonlinearity has a significant influence.

Let the control action for the stabilization of the pendulum be

$$
h=k_{1} \alpha+k_{2} \dot{\alpha} .
$$

Then the equation for $\alpha$ takes the form

$$
\left(\sin ^{2}(\epsilon-\alpha) \zeta+2\right) \ddot{\alpha}=(2+\zeta) \sin (\alpha)-\frac{1}{2} \zeta \dot{\alpha}^{2} \sin (2(\epsilon-\alpha))-2 \rho \cos (\epsilon-\alpha)\left(k_{1} \alpha+k_{2} \dot{\alpha}\right) .
$$


If we set $k_{2}=0$, then the equation of motion for the angle $\alpha$ has the conservation law

$$
\begin{aligned}
\mathcal{E}_{\epsilon}= & k_{1}\left(\rho\left(1+\frac{2}{\zeta}\right) \alpha^{2}+\frac{2}{\rho}(\cos (\epsilon-\alpha)-2 \alpha \sin (\epsilon-\alpha))\right)+ \\
& \cos (\alpha)(\zeta+2)+\sin (\epsilon) \sin (\epsilon-\alpha)+\left(\frac{1}{2} \sin ^{2}(\epsilon-\alpha) \zeta+1\right) \dot{\alpha}^{2} .
\end{aligned}
$$

The equation of motion turns out to be integrable by quadratures for $k_{2}=0$.

Stationary points for (5.3) satisfy the equation

$$
2\left((\zeta+2) \rho^{2}+\zeta \cos (\epsilon-\alpha)\right) k_{1} \alpha+\left((-\sin (\epsilon) \cos (\epsilon-\alpha)-2 \sin (\alpha)) \zeta-\sin (\alpha) \zeta^{2}\right) \rho=0 .
$$

Solutions to this equations in explicit form are not expressed in terms of elementary functions. For clarity of representation, it is convenient to rewrite this equation as

$$
k_{1} \alpha=\frac{(\sin (\alpha)(\zeta+2)+\sin (\epsilon) \cos (\epsilon-\alpha)) \zeta \rho}{2(\zeta+2) \rho^{2}+2 \cos (\epsilon-\alpha) \zeta} .
$$

This form of equation is convenient for graphical analysis. Figure 3 shows that, when $k_{1}=2$, there exist three points of intersection of the curve from the right-hand side of equation (5.5). For small values of $k_{1}$, for example, for $k_{1}=0.3$, there exists only one solution.

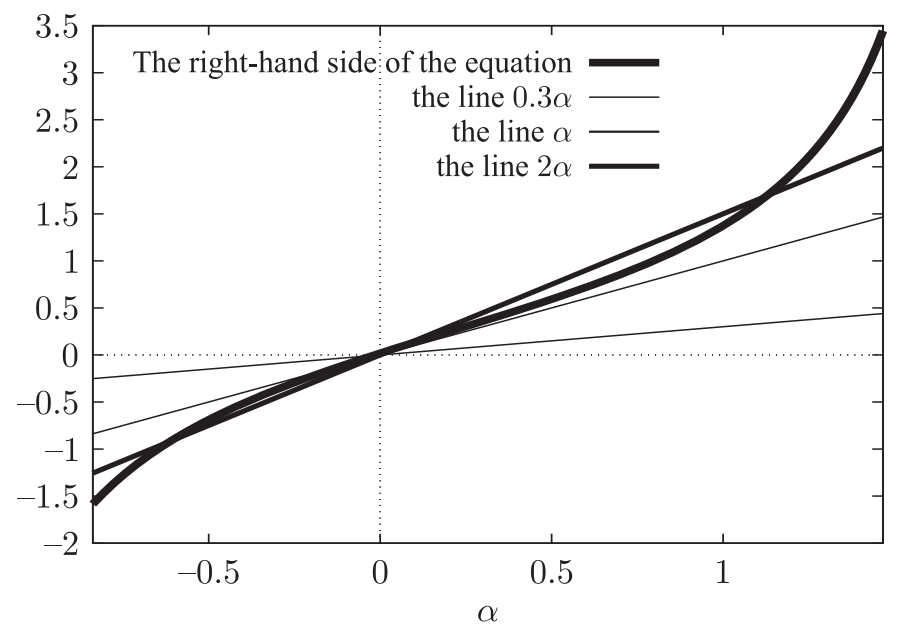

Fig. 3. The points of intersection of the heavy curve and the straight lines correspond to solutions of Eq. (5.5). The graph has been plotted for $\rho=0.2, \zeta=10, \epsilon=0.2$.

It can be shown that $\exists \epsilon_{0}, 0 \leqslant \epsilon_{0}<\pi / 2$, such that for the inclination angles $\epsilon \in\left[0, \epsilon_{0}\right)$ there exist values of the parameter $k_{1}$ such that for the equation for $\alpha \in(-\pi / 2, \pi / 2)$ there exist three stationary points $\alpha_{0,1,2}$ such that $-\pi / 2 \alpha_{1}<\alpha_{0}<\alpha_{2}<\pi / 2$. focus.

The points $\alpha_{1,2}$ are saddles on the phase plane $(\alpha, \dot{\alpha})$. When $k_{2}>0$, the point $\alpha_{0}$ is a stable

Since the integral $\mathcal{E}_{\epsilon}$ has different values at saddle points in the general position case, for each of the saddle points with $k_{2}=0$ there exist two homoclinic trajectories which tend to $\alpha_{i}$ as $t \rightarrow \pm \infty$. The stability region of the pendulum is defined by the smallest of the separatrix loops (see Fig. 4). 
The basin of stability for the inverted wheeled pendulum on the slanted plane

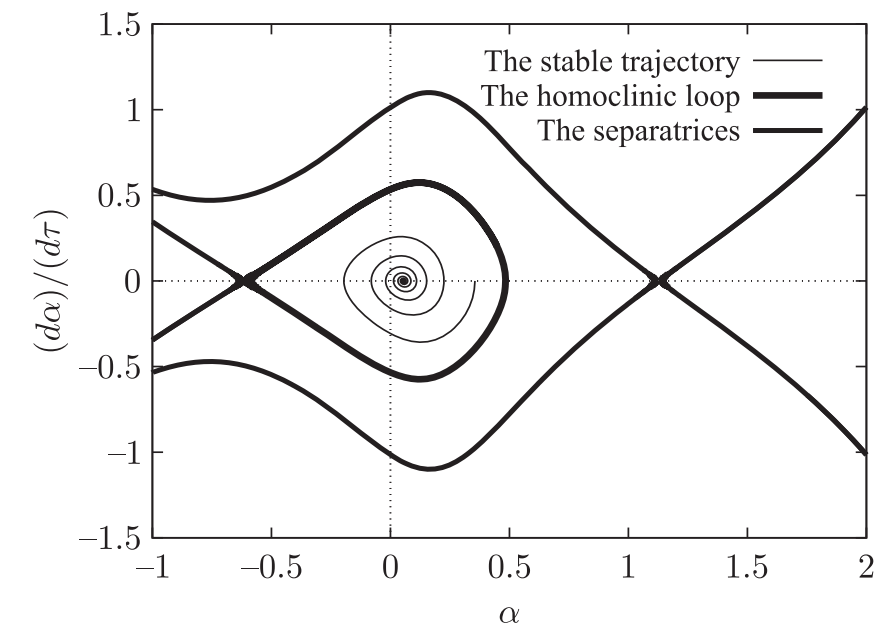

Fig. 4. Separatrices emanating from the left and right saddle points. The separatrix loop at the center defines the region of stable trajectories of the pendulum in a neighborhood of the point $(\alpha, 0)$. The curves have been plotted by numerically solving equation (5.1) by the Runge-Kutta fourth-order method for the parameter values: $\zeta=10, \rho=0.2, k_{1}=1.5$. For the trajectory converging to the point $\left(\alpha_{0}, 0\right)$, $k_{2}=0.05$.

Let us differentiate the function $\mathcal{E}_{\epsilon}$ by virtue of Eq. (5.1) with $k_{2}>0$. This yields

$$
\frac{d \mathcal{E}_{\epsilon}}{d \tau}=-2 k_{2} \dot{\alpha}^{2}\left(\left(1+\frac{2}{\zeta}\right) \rho+\frac{1}{\rho} \cos (\epsilon-\alpha)\right) \text {. }
$$

Denote

$$
f_{\epsilon}(\alpha, \zeta, \rho)=\left(\left(1+\frac{2}{\zeta}\right) \rho+\frac{1}{\rho} \cos (\epsilon-\alpha)\right)
$$

Then all trajectories inside the separatrix loop tend to the minimal value of $\mathcal{E}_{\epsilon}$, namely, to the point $\left(\alpha_{0}, 0\right)$, if the following condition is satisfied:

$$
f_{\epsilon}(\alpha, \zeta, \rho)>0, \quad \forall \alpha \in\left(\alpha_{1}, \alpha_{m}\right), \quad \alpha_{m}=\max _{\alpha \in \mathcal{L}}(\alpha), \quad \mathcal{L}=\left\{(\alpha, \dot{\alpha})\left|\mathcal{E}_{\epsilon}\right|_{\alpha=\alpha_{1}}\right\} .
$$

The main result of this section is the following theorem.

Theorem 2. Equation (5.1) has an asymptotically stable equilibrium point with coordinates $\left(\alpha_{0}, 0\right)$ on the phase plane provided that the function $h$ has the form (5.2) with coefficients $k_{1}$ and $k_{2}$ satisfying condition (5.6). Trajectories lying inside the separatrix loop defined by equation (4) with $\alpha=\alpha_{i}$, where $\left|\alpha_{i}\right|<\left|\alpha_{j}\right|, i, j=1,2$, tend to $\left(\alpha_{0}, 0\right)$ as $\tau \rightarrow \infty$. At the point $(\alpha, \dot{\alpha})=\left(\alpha_{0}, 0,\right)$ the acceleration of the wheel is $\ddot{\beta}=0$, i.e., the wheel can rotate with constant velocity.

\section{Stabilization of a pendulum on a soft surface}

During motion on a smooth soft surface the wheel gets into a hole and for incipient motion the torque must exceed some threshold value. Here we denote the coefficient, similar to the coefficient of rolling friction, by $\nu$.

For motion on a soft surface the following model is adopted.

If the wheel is not locked, then it is acted upon by the rolling friction torque $\nu \operatorname{sgn}(\dot{\beta})$. 
If the wheel is locked and the absolute value of the torque of the wheel is smaller than the value of $\nu$, then the rolling friction torque is unknown, but lies in the interval $(-\nu, \nu)$.

If during the lock-up of the wheel the absolute value of the wheel's torque becomes larger than the value of $\nu$, then the wheel begins to rotate and the whole inverted wheeled pendulum starts to move.

The motion on a horizontal soft surface is defined by the differential inclusion:

$$
\begin{gathered}
\ddot{\alpha}-\sin (\alpha)+2 \frac{\rho}{\zeta} h=\rho \cos (\alpha) \ddot{\beta}, \\
(\zeta+2) \rho \ddot{\beta} \in F(\alpha, \dot{\alpha}, \ddot{\alpha}, \dot{\beta}) .
\end{gathered}
$$

Denote

$$
f=-\left(\ddot{\alpha} \cos (\alpha)-\dot{\alpha}^{2} \sin (\alpha)\right) \zeta+\frac{2}{\rho} h .
$$

In (6.1) the map $F(\alpha, \dot{\alpha}, \ddot{\alpha}, \dot{\beta})$ has the following structure:

$$
F(\alpha, \dot{\alpha}, \ddot{\alpha}, \dot{\beta})=\left\{\begin{array}{cl}
f-\nu \operatorname{sgn}(\dot{\beta}), & \{\forall \dot{\beta} \neq 0\} ; \\
(-\nu, \nu), & \{\dot{\beta}=0\} \cup\{|f| \leqslant \nu\} ; \\
f-\nu \operatorname{sgn}(\dot{\beta}), & \{\dot{\beta}=0\} \cup\{\{\alpha, \dot{\alpha}, \ddot{\alpha}\} \in\{|f|>\nu\}\} .
\end{array}\right.
$$

Differential inclusions are often used to describe the motion with dry friction [18]. Rolling friction is an analog close to it. It is convenient to regard the differential inclusion (6.1) as two different dynamical systems: for the wheel lock mode and for the rotating wheel.

If the wheel is locked, $\dot{\beta}=0$, then $\beta=$ const. The equation for the deflection angle of the pendulum is

$$
\ddot{\alpha}-\sin (\alpha)+\frac{\rho}{\zeta}\left(k_{1} \alpha+k_{2} \dot{\alpha}\right)=0 .
$$

This equation can be regarded as an equation of the mathematical pendulum with dissipation and additional torque.

The obvious equilibrium position for Eq. $(6.2)$ is $(\alpha, \dot{\alpha})=(0,0)$. If $k_{1}>(\zeta / \rho)$, this is the only steady-state solution of the equation and this solution is stable.

If $k_{1}<(\zeta / \rho)$, the solution $(\alpha, \dot{\alpha})=(0,0)$ is unstable, and in the interval $\alpha \in(-\pi, \pi)$ there exist two more steady-state solutions to this equation $-\pi<\alpha_{1}<0<\alpha_{2}<\pi$. These solutions are stable foci when $k_{2}>0$.

If the values of $\alpha_{1}$ and $\alpha_{2}$ with $k_{1}<(\zeta / \rho)$ are such that $2 k_{1} \rho\left|\alpha_{1,2}\right| \leqslant \nu$, then there exist two stable stationary positions of the wheeled pendulum in which the wheel remains locked.

If the values of $\alpha_{1}$ and $\alpha_{2}$ with $k_{1}<(\zeta / \rho)$ are such that $2 k_{1} \rho\left|\alpha_{1,2}\right|>\nu$, then there exists no stable equilibrium position with the wheel locked.

This implies that in the case of a small deviation from the equilibrium position the deflection angle of the pendulum will begin to change and this change will lead to a change in the control torque of the wheel. The wheel will start to move.

Let us consider the dynamics of the pendulum on the segments of a trajectory with definite $\operatorname{sign} \operatorname{sgn}(\dot{\beta})$. The equation for $\alpha$ is

$$
\begin{aligned}
\left(2+\zeta \sin ^{2}(\alpha)\right) \ddot{\alpha}= & (2+\zeta) \sin (\alpha)-\frac{\zeta}{2} \dot{\alpha}^{2} \sin (2 \alpha)-2 \rho\left(1+\frac{2}{\zeta}\right)\left(k_{1} \alpha+k_{2} \dot{\alpha}\right)- \\
& \frac{2}{\rho} \cos (\alpha)\left(k_{1} \alpha+k_{2} \dot{\alpha}\right)+\operatorname{sgn}(\dot{\beta}) \nu \cos (\alpha) .
\end{aligned}
$$


On the segments with constant $\operatorname{sign} \operatorname{sgn}(\dot{\beta})$ and with $k_{2}=0$ this equation has the conservation law

$$
\begin{aligned}
\mathcal{E}_{\nu}= & \left(1+\frac{1}{2} \sin ^{2}(\alpha) \zeta\right) \dot{\alpha}^{2}+(\zeta+2) \cos (\alpha)-\nu \operatorname{sgn}(\dot{\beta}) \cos (\alpha)+ \\
& k_{1}\left(\frac{2}{\rho}(\alpha \sin (\alpha)+\cos (\alpha))+\alpha^{2} \rho\left(1+\frac{2}{\zeta}\right)\right) .
\end{aligned}
$$

If $k_{2} \neq 0$, then by virtue of this equation the derivative has the following form:

$$
\dot{\mathcal{E}}_{\nu}=-2 k_{2} \dot{\alpha}^{2}\left(\left(1+\frac{2}{\zeta}\right) \rho+\frac{1}{\rho} \cos (\alpha)\right) .
$$

If the wheel is not locked, then the equation for stationary positions of the pendulum has the form

$$
\frac{k_{1} \rho}{\zeta} \alpha=\frac{(\nu \operatorname{sgn}(\dot{\beta}) \cos (\alpha)+(\zeta+2) \sin (\alpha)) \rho^{2}}{2(\zeta+2) \rho^{2}+2 \cos (\alpha) \zeta}
$$

If this equation has three solutions for each of the cases $\operatorname{sgn}(\dot{\beta})= \pm 1-\pi / 2<\alpha_{1}^{ \pm}<\alpha_{0}^{ \pm}<$ $\alpha_{2}^{ \pm}<\pi / 2$, then at the point $\alpha_{0}^{ \pm}$the function $\mathcal{E}_{\nu}$ has a minimum. For the cases $\operatorname{sgn}(\dot{\beta})= \pm 1$ the minimum points of the function $\mathcal{E}_{\nu}$ are such that $\alpha_{0}^{-}<0<\alpha_{0}^{+}$.

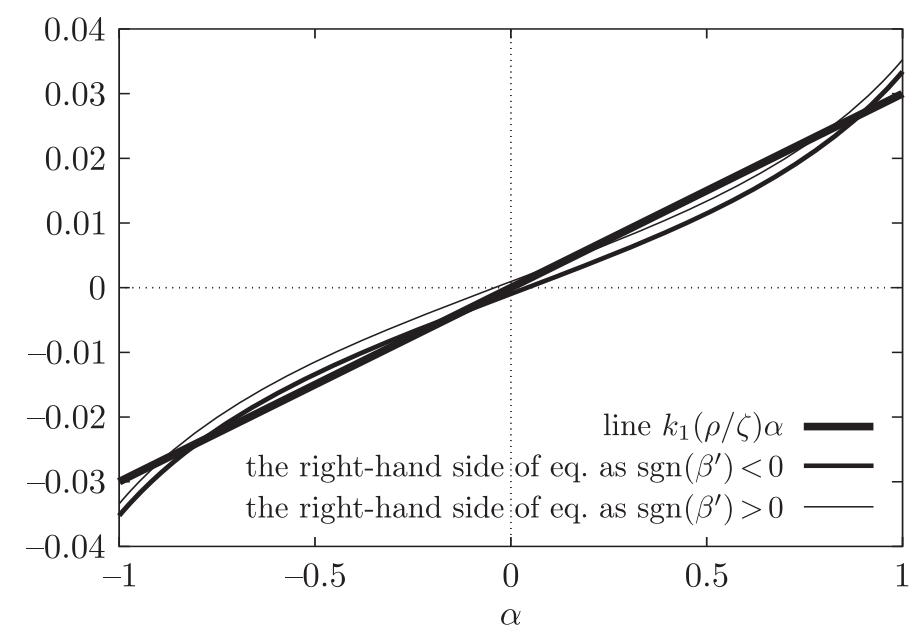

Fig. 5. The figure shows two curves corresponding to the right-hand sides of Eq. (6.3) with $\operatorname{sgn}(\dot{\beta})>0$ and $\operatorname{sgn}(\dot{\beta})>0$ for the values $\zeta=10, \rho=0.2, \nu=0.5$. The straight line corresponds to the the left-hand side of Eq. (6.3).

When the pendulum is stabilized at points $\alpha_{0}^{ \pm}$, under the condition $2 k_{2}\left|\alpha_{0}\right|>\rho \nu$, the acceleration of the pendulum's wheel is

$$
\ddot{\beta_{ \pm}}=\frac{1}{\rho(\zeta+2)}\left(\frac{2}{\rho} k_{1} \alpha_{0}^{ \pm}-\nu \operatorname{sgn}(\dot{\beta})\right)
$$

The constant acceleration of the wheel leads to an increase in the velocity of motion of the wheeled pendulum and eventually to the loss of stabilization when the maximal rotational speed is exceeded of which the motor rotating the wheel is capable. A numerical example for uniformly accelerated rotation of the wheel is given in Fig. 6 . 

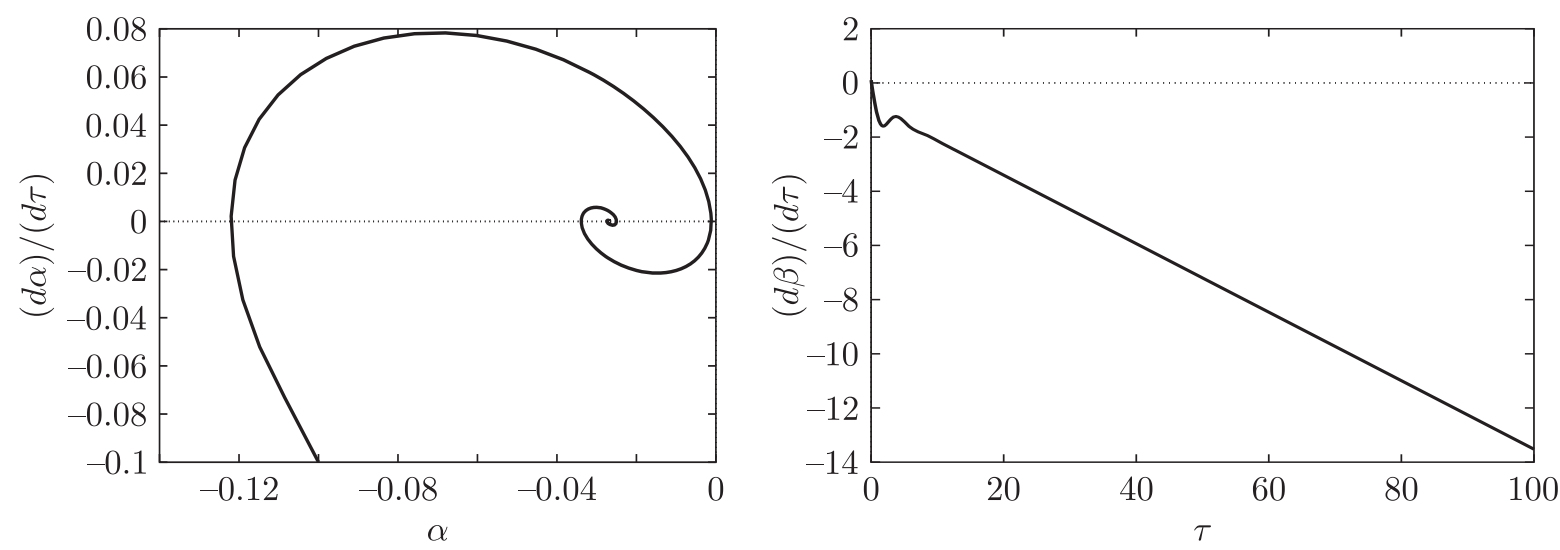

Fig. 6. The solution to Eqs. (6.1) with initial conditions $\tau=0, \alpha=-0.1, \dot{\alpha}=-0.1, \beta=0, \dot{\beta}=0.1$ and with a proportional-plus-derivative action control law, where $k_{1}=1.5, k_{2}=0.2$. The left figure shows the stabilization of the rotation angle of the pendulum $\alpha$, and the right figure shows the steady-state linear dependence of the rotation angle of the wheel $\beta$ on time $\tau$. The friction coefficient is $\nu=0.05$.

If $2 k_{2}\left|\alpha_{0}^{ \pm}\right| \leqslant \rho \nu$, then $\ddot{\beta}=0$ and the stabilization on $\alpha_{0}^{ \pm}$leads to the inequality

$$
-\nu<\frac{\zeta+2}{\cos \left(\alpha_{0}^{ \pm}\right)}\left(-\sin \left(\alpha_{0}^{ \pm}\right)+2 \frac{\rho}{\zeta} k_{1} \alpha_{0}^{ \pm}\right)<\nu
$$

Thus, for stabilization it suffices to construct a control that stabilizes the pendulum at the point $\alpha=0$ or in its small neighborhood.

To stabilize motion in this situation it suffices to introduce an integral component into the wheel's control system:

$$
h=k_{1} \alpha+k_{2} \dot{\alpha}+k_{3} \int_{0}^{\tau} \alpha(\theta) d \theta .
$$

As a result of introduction of the integral component into the control law, a term that increases linearly in absolute value for any constant values of $\alpha \neq 0$ is added to the autonomous equation of motion for $\alpha$. So that there exists the only steady-state solution $\alpha \equiv 0$. Indeed, let us denote

$$
A=\int_{0}^{\tau} \alpha d \theta
$$

Then the following third-order equation holds for $A$ :

$$
\begin{aligned}
\left(2+\zeta \sin ^{2}(\dot{A})\right) \dddot{A}= & (2+\zeta) \sin (\dot{A})-\frac{\zeta}{2} \ddot{A}^{2} \sin (2 \dot{A})+\operatorname{sgn}(\dot{\beta}) \nu \cos (\dot{A})- \\
& 2 \rho\left(1+\frac{2}{\zeta}\right)\left(k_{1} \dot{A}+k_{2} \ddot{A}+k_{3} A\right)-\frac{2}{\rho} \cos (\dot{A})\left(k_{1} \dot{A}+k_{2} \ddot{A}+k_{3} A\right) .
\end{aligned}
$$

The steady-state solution to this equation (with $\alpha=\dot{A} \equiv 0$ ) is

$$
A^{ \pm}=\frac{\operatorname{sgn}(\dot{\beta}) \zeta \nu \rho}{\left(2 k_{3} \zeta+4 k_{3}\right) \rho^{2}+2 k_{3} \zeta}
$$


Let us take the formula

$$
h=k_{1} \alpha+k_{2} \dot{\alpha}+A^{ \pm}+k_{3} \int_{\tau^{ \pm}}^{\tau} \alpha d \theta
$$

As a result, the equation for $\alpha$ with motion $\dot{\beta} \neq 0$ and with $\tau>\tau^{ \pm}$takes the form of an equation of motion along a horizontal line with an additional integral term:

$$
\begin{gathered}
\left(\sin ^{2}(\alpha) \zeta+2\right) \ddot{\alpha}=(\zeta+2) \sin (\alpha)-\frac{1}{2} \dot{\alpha}^{2} \zeta \sin (2 \alpha)- \\
2\left(\frac{1}{\rho} \cos (\alpha)+\left(1+\frac{2}{\zeta}\right) \rho\right)\left(k_{1} \alpha+k_{2} \dot{\alpha}+k_{3} \int_{\tau^{ \pm}}^{\tau} \alpha d \theta\right)
\end{gathered}
$$

When $k_{2}=k_{3}=0$, the conservation law coincides with the conservation law for the motion of the pendulum along the horizontal line:

$$
\mathcal{E}_{0}=\left(1+\frac{1}{2} \sin ^{2}(\alpha) \zeta\right) \dot{\alpha}^{2}+(\zeta+2) \cos (\alpha)+k_{1}\left(\frac{2}{\rho}\left(\alpha \sin (\alpha)+\cos (\alpha)+\alpha^{2} \rho\left(1+\frac{2}{\zeta}\right)\right) .\right.
$$

Let us differentiate $\mathcal{E}_{0}$ by virtue of Eq. (6.5) on the segment of motion without change of sign $\operatorname{sgn}(\dot{\beta})=$ const:

$$
\frac{d \mathcal{E}_{0}}{d \tau}=-2 k_{2}\left(\left(1+\frac{2}{\zeta}\right) \rho+\frac{1}{\rho} \cos (\alpha)\right)\left(\dot{\alpha}^{2}+\kappa_{3} \dot{\alpha} \int_{\tau^{ \pm}}^{\tau} \alpha(\theta) d \theta\right) .
$$

Here $\kappa_{3}=k_{3} / k_{2}$.

When $\kappa_{3}=0$, the motion coincides with the motion along the horizontal line, for which the statement of stability of the steady-state solution (Theorem 1) has been proved. When $\kappa_{3}>0$, the term with the integral on the right-hand side of the formula is not sign-definite. However, for sufficiently small values of $\kappa$ it may be expected that the integral addition will not lead to the loss of stability of the zero value of $\alpha$.

To investigate stability for small values of $\alpha$, we consider the linearized equation (6.5) in a neighborhood of $(\alpha, \dot{\alpha})=(0,0)$ :

$$
\ddot{a}=-k_{3} s \int_{\tau^{ \pm}}^{\tau} a(\theta) d \theta-k_{2} s \dot{a}-\left(s-\frac{\zeta}{2}-1\right) a,
$$

where

$$
s=\left(\frac{2 \rho}{\zeta}+\rho+\frac{1}{\rho}\right)
$$

Let us differentiate this equation with respect to $\tau$. Then the characteristic equation for the third-order equation is

$$
\lambda^{3}=-k_{3} s-k_{2} s \lambda^{2}-\left(s-\frac{\zeta}{2}-1\right) \lambda .
$$

Denote the solutions to this equation by $\lambda_{j}, j=1,2,3$. In the general position case the stability condition for $(\alpha, \dot{\alpha})=(0,0)$ is

$$
\operatorname{Re}\left(\lambda_{j}\right)<0, \quad j=1,2,3 .
$$


If we consider small values of $k_{2}$ and $k_{3}$, then the stability condition takes the form

$$
\left(\frac{2 \rho}{\zeta}+\rho+\frac{1}{\rho}\right) k_{1}>\frac{\zeta}{2}+1, \quad 0<k_{3}<k_{2}\left(\frac{2 \rho}{\zeta}+\rho+\frac{1}{\rho}\right) .
$$

Let us formulate the main statement.

Theorem 3. There exist $k_{2}^{0}>0$ and $k_{3}^{0}>0$ such that Eq. (6.5) in the interval $\operatorname{sgn}(\dot{\beta})=$ $=$ const has an asymptotically stable equilibrium point with coordinates $\left(\alpha_{0}, 0\right)$ on the phase plane under condition (6.6), where $k_{2}<k_{2}^{0}$ and $k_{3}<k_{3}^{0}$.
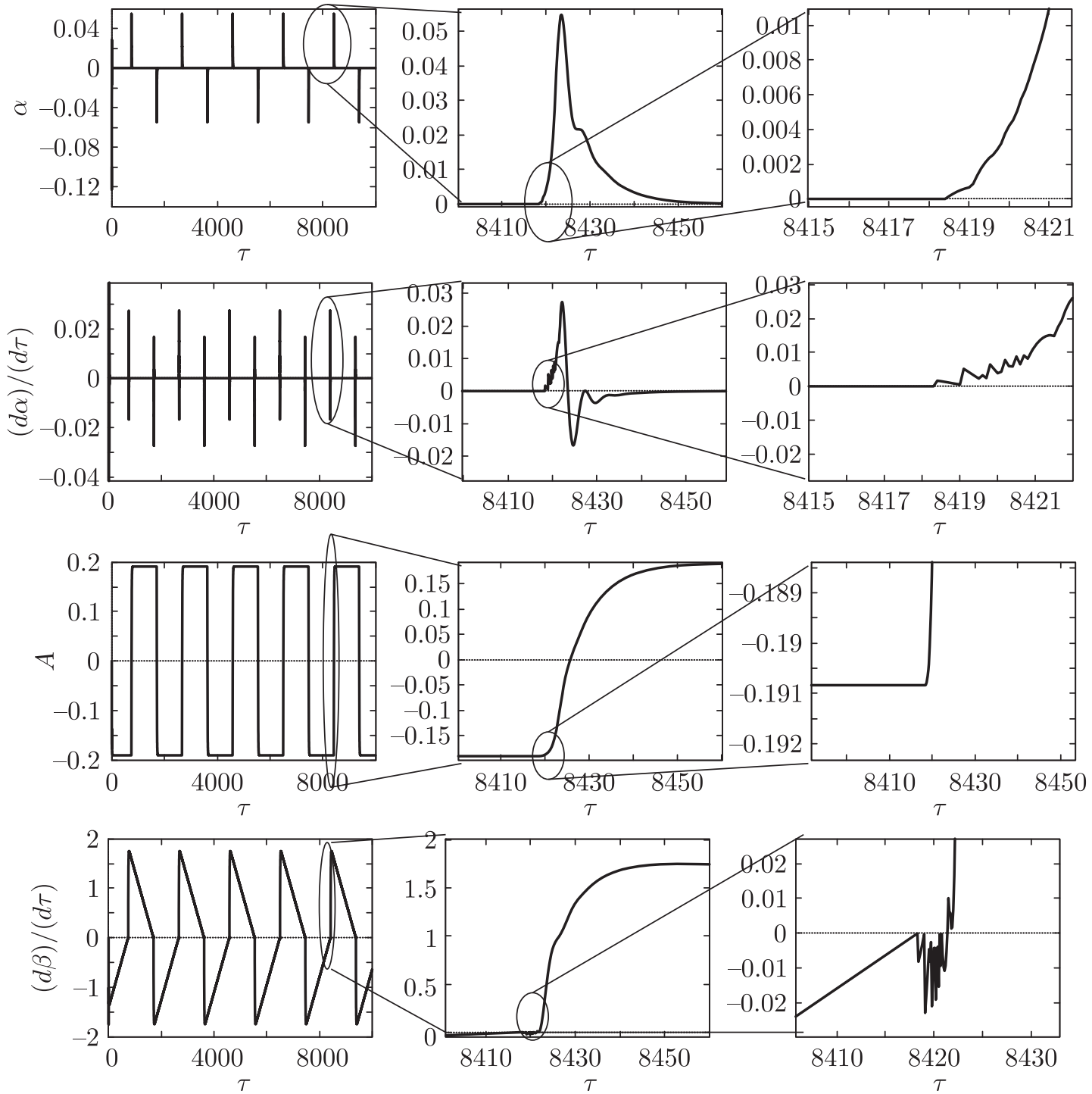

Fig. 7. A solution to (6.1) with initial conditions $\tau=0, \alpha=-0.1, \dot{\alpha}=-0.1, \beta=0, \dot{\beta}=0$ and with a proportional plus integral plus derivative action control law, where $k_{1}=1.5, k_{2}=0.2, k_{3}=0.05$. The upper panels show the stabilization of the rotation angle of the pendulum $\alpha$; the row of panels second from the top shows the rate of change in the rotation angle $\dot{\alpha}$; the row of panels third from the top shows a graph of the value of the integral component in the controller $A=\int_{0}^{\tau} \alpha(\theta) d \theta$; the lower row of panels shows the velocity of the wheel $\dot{\beta}(\tau)$. The parameters of the pendulum are $\zeta=10, \rho=0.2$, and the friction coefficient is $\nu=0.1$. 
Theorem 3 provides a qualitative description of the dynamics of a wheeled pendulum controlled by a PID controller. Typical stages are presented in Fig. 7, which illustrates calculation results for a wheeled pendulum on a soft surface. In particular, the row of graphs third from the top shows how fast the integral component of the controller increases. Then it stabilizes until the rotational velocity of the wheel $\dot{\beta}$ changes sign. In the interval with a stable value of the integral component one has $(\alpha, \dot{\alpha}) \rightarrow(0,0)$ and $\dot{\beta} \rightarrow-0$.

The limiting value $A=A^{-}, \alpha=0, \dot{\alpha}=0, \dot{\beta}=0$ is a steady-state solution which is stable below, but unstable above when $\dot{\beta}=0+\delta \forall \delta>0$. With the perturbations present in the real system and with the round-off errors generated in the computational scheme this leads eventually to a change of the sign of $\dot{\beta}$.

After the change of the sign of $\dot{\beta}$ the system rearranges itself fast. The integral component stabilizes at the new value $A=A^{+}$. The process of stabilization repeats itself: $(\alpha, \dot{\alpha}) \rightarrow(0,0)$ and $\dot{\beta} \rightarrow+0$. In this case, the limiting solution $A=A^{+}, \alpha=0, \dot{\alpha}=0, \dot{\beta}=0$ is a steady-state solution stable above, but unstable below. With the passage of time, due to perturbations in the real system or round-off errors in the numerical scheme, $\dot{\beta}$ eventually changes sign and the process repeats itself. This gives rise to a limit cycle with a period depending on a random quantity - perturbations in the real system or a pseudo-random variable - a round-off error in the numerical scheme.

Figure 8 shows projections of trajectories onto the phase planes $(\alpha, \dot{\alpha})$ and $(\beta, \dot{\beta})$.
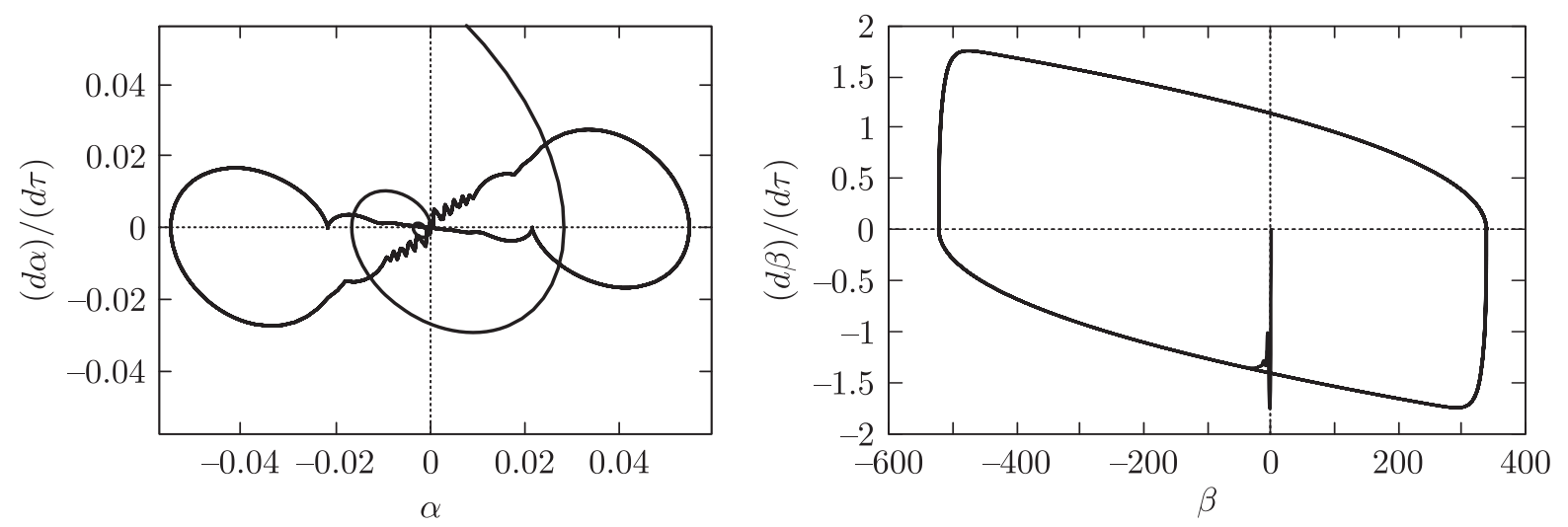

Fig. 8. A solution to (6.1) with initial conditions $\tau=0, \alpha=-0.1, \dot{\alpha}=-0.1, \beta=0, \dot{\beta}=-0.1$ and with a proportional plus integral plus derivative action control law, where $k_{1}=1.5, k_{2}=0.2, k_{3}=0.05$. The figure shows a limit cycle for the rotation angle of the pendulum $\alpha$, the right panel shows a stable limit cycle on the phase plane $(\beta, \dot{\beta})$. The parameters of the pendulum are $\zeta=10, \rho=0.2$, and the friction coefficient is $\nu=0.1$.

\section{Conclusion}

In this paper it is shown that for stabilization of the wheeled pendulum on a smooth and inclined surface it suffices to use a proportional plus derivative action controller.

For stabilization of the wheeled pendulum on a soft surface, the proportional plus derivative action controller can stabilize the pendulum in the upper position, but in this case the wheel moves with constant acceleration. To stabilize the rotational velocity of the wheel, it is proposed to use the proportional plus integral plus derivative action controller. It turns out that stabilization on a soft surface occurs on the limit cycle near the state of unstable equilibrium of the pendulum. 


\section{References}

[1] Andrievsky, B.R. and Fradkov, A.L., Selected Chapters of Control Theory with Examples in MATLAB, St. Petersburg: Nauka, 1999 (Russian).

[2] Åström, K. J., Block D. J., and Spong M.W., The Reaction Wheel Pendulum, San Rafael, Calif.: Morgan \& Claypool, 2007.

[3] Halkyard, C. R., Chan, R. P. M., and Stol, K. A., Review of Modelling and Control of Two-Wheeled Robots, Annu. Rev. Control, 2013, vol.37, no. 1, pp. 89-103.

[4] Formalskii, A. M., Stabilization and Motion Control of Unstable Objects, Stud. Math. Phys., vol. 33, Berlin: de Gruyter, 2015.

[5] Martynenko, Yu. G. and Formal'skii, A. M., Controlled Pendulum on a Movable Base, Mech. Solids, 2013, vol.48, no.1, pp.6-18; see also: Izv. Akad. Nauk. Mekh. Tverd. Tela, 2013, no. 1, pp.9-23.

[6] Colombi, S., Grasser, F., D’Arrigo, A., and Rufer, A. C., Joe: A Mobile, Inverted Pendulum, IEEE Trans. Ind. Electron., 2002, vol. 49, no. 1, pp. 107-114.

[7] Pathak, K., Franch, J., and Agrawal, S. K., Velocity and Position Control of a Wheeled Inverted Pendulum by Partial Feedback Linearization, IEEE Trans. Robot., 2005, vol. 21, no. 3, pp. 505-513.

[8] Francovský, P., Dominik, L., Gmiterko, A., Virgala, I., Kurylo, P., and Perminova, O., Modeling of Two-Wheeled Self-Balancing Robot Driven by DC Gearmotors, Int. J. Appl. Mech. Eng., 2017, vol. 22, no. 3, pp. 739-747.

[9] Nasrallah, D. S., Michalska, L., and Angeles, J., Controllability and Posture Control of a Wheeled Pendulum Moving on an Inclined Plane, IEEE Trans. Robot., 2007, vol. 23, no. 3, pp. 564-577.

[10] Kunz, T., Teeyapan, K., Wang, J., and Stilman M., Robot Limbo: Optimized Planning and Control for Dynamically Stable Robots under Vertical Obstacles, in IEEE Int. Conf. Robot. Autom. (Anchorage, Alaska, May 3-8, 2010), pp. 4519-4524.

[11] Stol, K., Kausar, Z., and Patel, N., Performance Enhancement of a Statically Unstable Two Wheeled Mobile Robot Traversing on an Uneven Surface, in 2010 IEEE Conference on Robotics Automation and Mechatronics (RAM), pp. 156-162.

[12] Stol, K., Kausar, Z., and Patel, N., The Effect of Terrain Inclination on Performance and the Stability Region of Two-Wheeled Mobile Robots, Int. J. Adv. Robot. Syst., 2012, vol. 9, no. 5, 11 pp.

[13] Åström, K. J. and Hägglund, T., PID Controllers: Theory, Design, and Tuning, 2nd ed., 1994.

[14] Kiselev, O. M., Mathematical Foundations of Robotics, Oryol: Kartush, 2019.

[15] Shimada, A. and Hatakeyama, N., Movement Control Using Zero Dynamics of Two-Wheeled Inverted Pendulum Robot, in 10th IEEE International Workshop on Advanced Motion Control (Trento, Italy, March 26-28, 2008), pp. 38-43.

[16] Ahmad, M. A., Nasir, A. N. K., and Raja Ismail, R. M.,T., Performance Comparison between Sliding Mode Control (SMC) and PD-PID Controllers for a Nonlinear Inverted Pendulum System, in 11th Internat. Conf. on Control, Automation, Robotics and Vision (ICARCV 2010), pp. 122-127.

[17] Maddahi, A., Shamekhi, A. H., and Ghaffari, A., A Lyapunov Controller for Self-Balancing TwoWheeled Vehicles, Robotica, 2015, vol.33, no. 1, pp. 225-239.

[18] Filippov, A.F., Differential Equations with Discontinuous Righthand Sides, Math. Appl., vol. 18, Dordrecht: Springer, 1988. 